\title{
Skeleton based Signatures for Content based Image Retrieval
}

\author{
M. Narayana \\ Jaya Prakash Narayan \\ College of Engineering, \\ Mahabubnagar, A.P., India
}

\author{
Sandeep V.M \\ Jaya Prakash Narayan \\ College of Engineering \\ Mahabubnagar, A.P., India
}

\author{
Subhash Kulkarni \\ Jaya Prakash Narayan \\ College of Engineering \\ Mahabubnagar,A.P., India
}

\begin{abstract}
Content Based Image Retrieval with fast and high matching retrieving ability is the need of the day for shape mining. A simple, fast, robust, invariant and efficient Content Based image Retrieval system with shape signatures derived from skeleton, region and boundary of the object is presented. The shape signatures derived from distance mapped function are invariant to rotation and scaling.
\end{abstract}

\section{Keywords}

Skeleton; Shape Signature; Shape Retrieval; CBIR; DSFT.

\section{INTRODUCTION}

In recent years, there has been a growing interest in finding similar images from remote and widely spread database on the Internet. Content-based image retrieval (CBIR) architecture is one, which automatically searches images relevant to a query image from the Web or large image datasets using the inherent characteristics of image itself. The effectiveness of retrieval is dependent on two main issues: i) finding effective features, which encode maximum image content and ii) quantifying these features towards efficient similarity measurements. Object shape has been an important feature in searching images containing the object.

The shape signatures for object recognition proposed in this paper are obtained from the Skeleton, region, and boundary information. These signatures possess unique ability to distinguish slightest difference between shapes. Skeletons are primarily region based geometric descriptor for shape classification and CBIR applications. The skeleton signatures discussed in this paper (shape representation) are invariant to rotation, scaling, translation.

Distance mapped level set function, defined by ChanVese, is a powerful tool to separate out the object of interest from its background. For fast segmentation, the level set may be defined by city-block distance metric through the fast and robust Distance mapping with Scanning and Filling Technique (DSFT) [12]. At the steady state, the level set is a city-block distance map with the object and its background differentiated by the sign. In this work, distance map is used to extract the skeleton for the object under watch $[2,7,15]$.

The following section presents needed background in existing literature. In Section3, discusses the Proposed Architecture for CBIR with skeleton signatures. In section4, we present simple, fast and efficient methodology for extraction of skeleton and the shape signatures. Section5 presents the algorithm proposed. Justification through qualitative and quantitative results are presented in section6. Section7 concludes the paper with future direction for the proposed work.

\section{BACKGROUND}

The feature signatures in CBIR with fast matching and high retrieving ability are the need of the day for shape mining. In this section evaluation of reduced computational complexity and better retrieval efficiency have been discussed and compared amongst recent similar works.

CBIR based on Shape Descriptors such as Fourier descriptor (FD), curvature scale space descriptors (CSSD), Zernike moment descriptors (ZMD), Grid descriptors (GD), patch-based feature, and Generic segments(GSs) have been reported in recent literature $[4,18,19,20]$. The performance of these shape descriptors are presented in terms of Precision and Recall. On the contrary all the four methods tend to exhibit relatively low Precision with increase in database volume.

Obtaining accurate skeleton in less time is one of the emerging research areas. Skeleton (or medial axis) which integrates geometrical and topological features of the object is the most relevant shape descriptor for object recognition. Shape similarity based on skeletons suffers from certain instability issues and matching of skeleton graphs is still an open problem $[3,5,9,12,16]$.

Skeleton Extraction suggested by authors in [9] is time consuming and complex, an adaptive snake is used to extract the objects contour. On the contrary in [17] authors preferred to use curve evolution along with edge-strength calculated from grayscale images which is certainly time consuming. Skeleton extraction from graphs and identifying the skeleton nodes are difficult and time consuming techniques as suggested in skeleton based shape classification $[1,6,10,20]$.

In this paper, Active Contours without Edges proposed by Chan-Vase to segment and detect the boundary of the object is used. This model best detects boundary of the object and even works well with precision in the natural images $[2,7,13]$. The proposed method based on Skeleton Signatures have been found to exhibits better performance with reduced computational complexity and improved retrieval efficiency as compared to Contourlet transforms features, polar raster sampling(PRS), and area-ratio(AR) methods[20].

\section{PROPOSED ARCHITECTURE FOR CBIR WITH SKELETON SIGNATURE}

The proposed work is an extension of our earlier work [15]. The earlier process has been useful as a coarse level preprocessing step in hierarchical CBIR that reduces the database from very large to relatively small volume. The 
objective of the proposed work in this paper is to study the skeleton based shape signatures as effective features for CBIR. CBIR system retrieves the relevant shapes from the shape database for the given query shape by computing the signature features of the query shape and comparing with similar feature set of corresponding shapes in the database. Relevant shapes having minimum distance (or maximum similarity) computed between features of query shape and feature set in shape database are retrieved. In building a
CBIR system, two foundational issues need to be addressed.

1. Every shape in database has to be represented efficiently with unique significant optimum features.

2. The shape features should guarantee maximum number of relevant shape extraction from database with least time and space complexity.

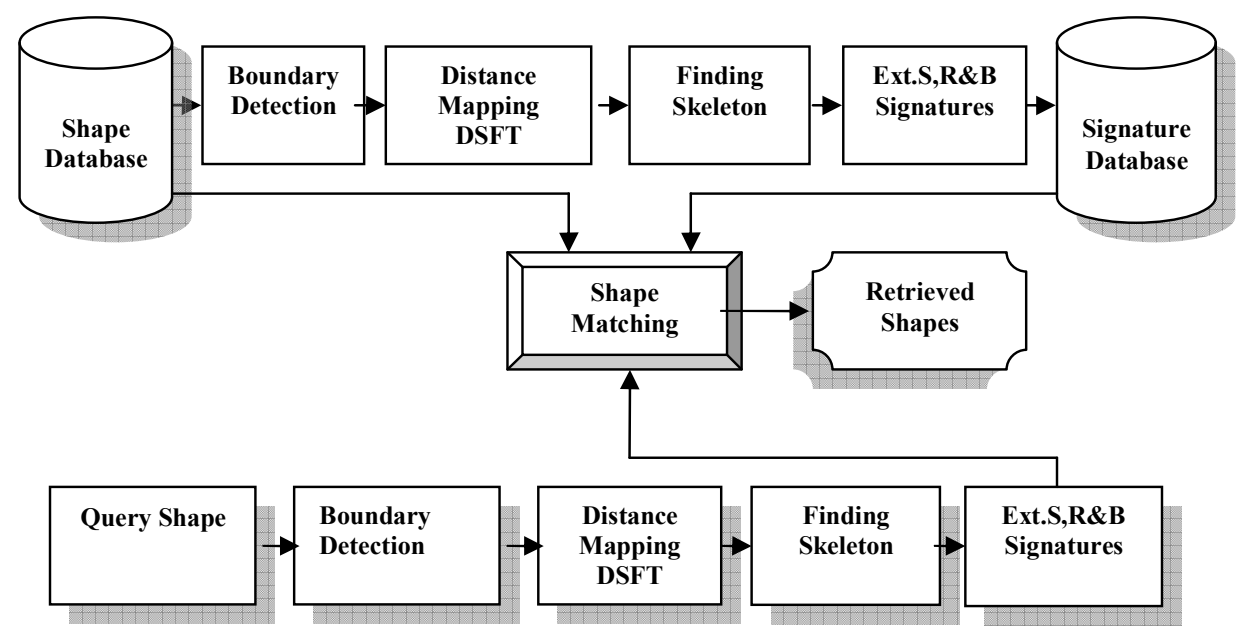

Fig 1: Proposed CBIR Architecture

In this regard, we propose a system where in the uniqueness of skeleton of the shape is used as the primary feature. This system uses the size of the skeleton rather than its shape in defining the shape signature and hence is fast and efficient when compared to other techniques. This simplicity of the feature computation is the major strength and advantage of the proposed system.

Fig1 presents the proposed CBIR architecture that can perform better shape mining. In the proposed architecture the signature database building is the primary step. The efficiency of system relies on building an effective and unique feature database. The process starts with the application of level set $[3,17,18,25]$ to segment the object out of the image. The level set is defined through 'Distance mapping using Scanning and Filling Technique' (DSFT) [12] whose time complexity is invariant to the size of initial curve and hence random initialization ensures fast and efficient segmentation. The segmented result is a zero valued level curve within level set function representing the boundary of the object. This distance mapped result of segmentation process encouraged us to use it to extract the skeleton and region signatures along with the boundary for building the shape signature database.

The performance of the proposed CBIR system is tested by retrieving the specified number of shapes from the database. The average retrieval rate and retrieval time are the main performance measures in the proposed CBIR system. The average retrieval rate is known as the percentage average number of shapes belonging to the same image as the test (query) shape in the top ' $\mathrm{N}$ ' matches. ' $\mathrm{N}$ ' indicates the number of retrieved shapes. Thus satisfying the $2^{\text {nd }}$ issue needed in CBIR system.

\section{SHAPE SIGNATURES FROM SKELETON}

We propose significant and effective feature signatures for the shape to improve the speed and efficiency of the object recognition process. The feature extraction phase is always preceded by segmentation process to separate out the object under consideration from its background. We use chan-vese model of level set for this purpose due to its higher speed and accuracy. The level set is defined by cityblock distance mapping through DSFT that is very fast and invariant to the size of the initial curve, providing a function mapped with city-block distances for the feature extraction phase. Hence, it makes sense to use this available data to extract the skeleton for the given shape.

\subsection{Skeleton Extraction}

Skeletoniztion is a process for reducing foreground regions in a binary image to a skeletal remnant that largely preserves the extent and connectivity of the original region while throwing away most of the original foreground pixels. This paper highlights the power of city-block distance map in skeletonization of a given shape.

The readily available city-block distance map from the level set based segmentation encouraged us to do so. In turn, DSFT (Distance mapping using Scanning and Filling Technique), was used to map the distances with city-block metric with assured high speed and robustness. This fast approach of segmenting and skeletonizing the object motivated to undertake the present work.

Fig 2 represents the distance mapping for a square shape, represented with zeros, city-block metric and the skeleton points are highlighted. This paper aims at extracting the skeleton points from this distance-mapped function. 
It is very clear, from the Fig 2 that the change in slope of the distance map corresponds to the skeleton points. This property of distance map encourages one to easily extract the skeleton points simply by searching the points of slope deviation. The skeleton of an object can be extracted by scanning the distance map function for row wise and column wise through forward or backward distances. For example, with backward distances, a point $(i, j)$ can be a skeleton point if one or both of the following conditions are true.

$D(i, j-1)-D(i, j) \neq D(i, j)-D(i, j+1)$

and/or

$D(i-1, j)-D(i, j) \neq D(i, j)-D(i+1, j)$

\subsection{Skeleton based shape signatures for CBIR}

We propose significant and effective feature signatures for the shape of the object through the skeleton.
The proposed two signatures uniquely distinguish between different shapes. The shape signatures are robust to scaling, rotation, and position and are very precise. These signatures are primarily an integration of region, boundary, and convexity of the objects in images.

The shape signature proposed through Skeletonization is obtained from a simple City-block distance mapping using Scanning and Filling Technique (DSFT)[12,13,14]. As mentioned obtaining Skeleton at faster rate is still an open problem. The DSFT technique involves only scanning through the rows, columns and updating each grid with a counter, which is independent of convolution, hence, is a fast method.

The proposed two skeleton signatures are derived from shape region and boundary.

- The first skeleton based region signature is defined as ratio of number of points on the skeleton and number of points inside the object.

- The second signature is defined as ratio of number of points on the skeleton and number of points on the boundary of the object.

\begin{tabular}{|l|l|l|l|l|l|l|l|l|l|l|}
\hline 0 & 0 & 0 & 0 & 0 & 0 & 0 & 0 & 0 & 0 & 0 \\
\hline 0 & 1 & 1 & 1 & 1 & 1 & 1 & 1 & 1 & 1 & 0 \\
\hline 0 & 1 & 2 & 2 & 2 & 2 & 2 & 2 & 2 & 1 & 0 \\
\hline 0 & 1 & 2 & 3 & 3 & 3 & 3 & 3 & 2 & 1 & 0 \\
\hline 0 & 1 & 2 & 3 & 4 & 4 & 4 & 3 & 2 & 1 & 0 \\
\hline 0 & 1 & 2 & 3 & 4 & 5 & 4 & 3 & 2 & 1 & 0 \\
\hline 0 & 1 & 2 & 3 & 4 & 4 & 4 & 3 & 2 & 1 & 0 \\
\hline 0 & 1 & 2 & 3 & 3 & 3 & 3 & 3 & 2 & 1 & 0 \\
\hline 0 & 1 & 2 & 2 & 2 & 2 & 2 & 2 & 2 & 1 & 0 \\
\hline 0 & 1 & 1 & 1 & 1 & 1 & 1 & 1 & 1 & 1 & 0 \\
\hline 0 & 0 & 0 & 0 & 0 & 0 & 0 & 0 & 0 & 0 & 0 \\
\hline
\end{tabular}

Fig 2: Distance mapped points on 11X11 Square grid

The procedures for shape signatures extraction from the skeleton, region, and boundary of the object is given below. The relationship used in this paper amongst the number of points having values on the skeleton, inside object, and on the boundary are the main features used to distinguish between different shapes as described below.

1. Skeleton based region signature of the given different shapes in the shape database given by
$S_{R}^{T}$ is the ratio of number of points on the skeleton and number of points inside the shape, denoted as $S_{R}^{T}=\frac{N_{S}}{N_{I}}$

2. Skeleton based boundary signature of the given different shapes in the shape database given by 
$S_{B}^{T}$ is the ratio of number of points on the skeleton and number of points on the boundary of shape,

$$
\text { denoted as } S_{B}^{T}=\frac{N_{S}}{N_{B}}
$$

3. For query shape, the shape signatures as given in above steps are denoted as

$$
\begin{aligned}
& S_{R}^{Q}=\frac{N_{S}}{N_{I}} \\
& S_{B}^{Q}=\frac{N_{S}}{N_{B}}
\end{aligned}
$$

4. The similarity measure between features using Euclidean distance metric is given as

$$
D_{E}=\sqrt{\left(S_{R}^{T}-S_{R}^{Q}\right)^{2}+\left(S_{B}^{T}-S_{B}^{Q}\right)^{2}}
$$

Where $D_{E}$ is the Euclidean Distance between the shape signature of the Query shape and individual shape in the database.

$$
S_{R}^{T}=\text { Skeleton based region signature of training }
$$
shapes

$S_{B}^{T}=$ Skeleton based boundary signature of training shapes

$S_{R}^{Q}=$ Skeleton based region signature of query shape

$$
S_{B}^{Q}=\text { skeleton based boundary signature of query shape }
$$

\section{PROPOSED ALGORITHM}

The basic steps involved in the proposed CBIR system include database processing and creation of signature database, comparison and shape retrieval. Sequential steps of the proposed algorithm are as follows.

1. Boundary detection using Chan-Vase level set model

2. Distance Mapped level set function building with boundary information

3. Skeleton extraction using slope change criteria

4. Compute skeleton based region signature of the given different shapes in the shape database using equation (1)

5. Compute skeleton based boundary signature of the given different shapes in the shape database using equation (2)

6. For test (query) shape calculate the shape signature as given in steps 1 to 5 and using equations (3) and (4).

7. Calculate the similarity measure using Euclidean distance using equation (5).
8. Retrieve all relevant shapes to test shape based on minimum distance between the features set.

The Euclidean distance between training shapes and query shape is computed. The distances are then sorted in increasing order and the top 20 nearest sets of shapes are then retrieved and are used for computing the performance of the proposed method. The retrieval (matching) efficiency has been measured by Precision and Recall performance.

\section{RESULTS AND DISCUSSIONS}

In this work, skeleton extraction of the proposed method is compared with three other methods of skeletonization, two of which are based on the maximal disk approach and third method uses contouring by snake. These three methods are iterative and consume more time to extract skeletons. Our method is non-iterative, fast, and robust. Hence proposed method is superior to above three methods.

Matching (retrieval) performance in terms of Precision and Recall and retrieval time of the proposed CBIR system has been tested by conducting experiment on signature database. recision \& Recall have been computed as Precision $=$ No. of relevant shapes retrieved (r)/Total No. of shapes retrieved $(\mathrm{n})$, Recall $=$ No. of relevant shapes retrieved (r)/Total No. of relevant shapes in the whole database $(\mathrm{m})$.

$$
P=\frac{r}{n} \quad R=\frac{r}{m}
$$

Precision (P) indicates the accuracy of the retrieval and the Recall (R) indicates the robustness of the retrieval performance. For each query, the precision of the retrieval at different levels of the recall are obtained. By selecting query shapes from the Database, the system was tested for top 5, top 10, top 15, and top 20-retrieved shapes, and the results have been shown in Table 1 . The database consists of 27 shapes with 20 instances of each shape making 540 images. The paper emphasizes more on shape signature and hence the database used for this work is the postsegmentation process i.e., binary images.

Normally, precision reduces as recall goes up, due to the fact that the system attempts to retrieve all relevant items to a query and in the process, some irrelevant items are also retrieved, thus reducing the precision. Conversely, the higher the precision, the lower the recall as the system filters off much of the shapes and many relevant items remain unretrieved. This can be observed for duck and leaf shapes in the Table 1. Thus a trade off is needed between these two measures since improving one will sacrifice the other. In practice, a precision-recall (PR) curve as shown in Fig 3 can be used to reflect this trade-off. In this method, precision of some of the shapes remains high as recall increases, which is very much significant and characteristic in our proposed method. This can be observed from Table 1. Our proposed method outperforms over the recently proposed shape signature methods in literature [20]. 
Table1: Precision and Recall values of the proposed system in percentage

\begin{tabular}{|l|l|l|l|l|l|l|l|l|}
\hline \multirow{2}{*}{$\begin{array}{l}\text { Query } \\
\text { Shape }\end{array}$} & \multicolumn{2}{|c|}{$\mathrm{n}=5$} & \multicolumn{2}{c|}{$\mathrm{n}=10$} & \multicolumn{2}{c|}{$\mathrm{n}=15$} & \multicolumn{2}{c|}{$\mathrm{n}=20$} \\
\cline { 2 - 9 } & Precision & Recall & Precision & Recall & Precision & Recall & Precision & Recall \\
\hline Apple & 100 & 25 & 100 & 50 & 100 & 75 & 100 & 100 \\
\hline Elephant & 100 & 25 & 100 & 50 & 100 & 75 & 95 & 95 \\
\hline Snake & 100 & 25 & 100 & 50 & 100 & 75 & 90 & 90 \\
\hline Duck & 100 & 25 & 100 & 50 & 86 & 80 & 80 & 80 \\
\hline Leaf & 100 & 25 & 90 & 45 & 80 & 60 & 75 & 75 \\
\hline
\end{tabular}

Where ' $n$ ' is number of shapes retrieved with 20 relevant shapes present in the database

The retrieval performance and accuracy results are based on Precision and Recall plots of shapes in the proposed skeleton based signatures (skl-sig) method and is compared with polar raster sampling (PRS) and area-ratio (AR) methods [20]. For each query, the precision of the retrieval at the level of recall is obtained. The precision and recall plots of the retrieval are shown in Fig 3. As can be observed from the graphs the proposed skeleton-based signatures for CBIR has superior performance over other two shape descriptors.

In Table 1, duck, and leaf shapes, retrieval performance with proposed method is not satisfactory because these shapes possess concave curvatures around the boundary of the shape. The proposed shape signatures pose limitations in describing concave curvature shapes. Hence, the percentage of precision and recall falls between 70 and $75 \%$. For other shapes the percentage of precision and recall are very good and are about 90 to $100 \%$ as shown in Table 1 , which have very little or no concavity.

Fig 4 and 5 show the top 20 shapes retrieved for elephant and Leaf shapes respectively.

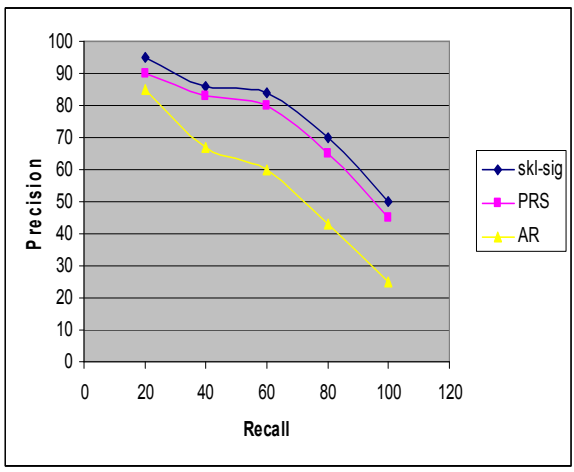

Fig 3: Average Retrieval Performance

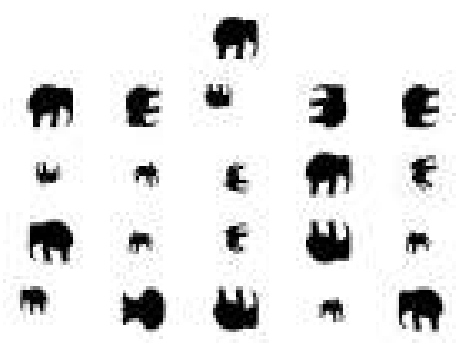

Fig 4: Shows results of Elephant shape retrieval and top 20 matches

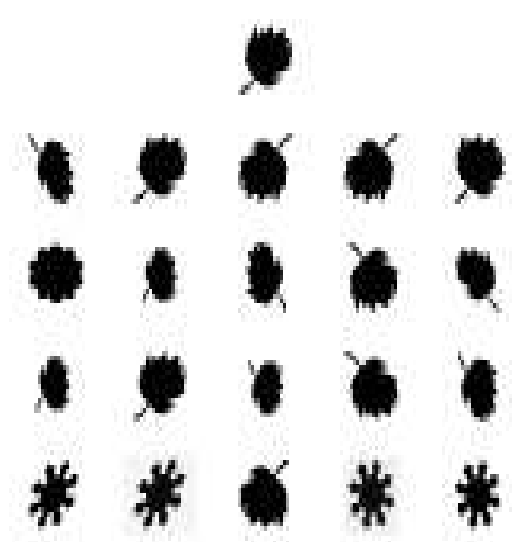

Fig 5: Shows results of Leaf shape retrieval and top 20 matches 


\section{CONCLUSION AND FUTURE SCOPE}

In this paper, a novel fast, efficient and

Simple method of deriving the shape signatures using the skeleton is presented. Superiority of this work has been observed in terms of reduced computation time as compared to other methods.

We have proposed system architecture for the Content Based Image Retrieval by Skeleton derived Region and Boundary shape signatures. The retrieval performance of the proposed method is presented in Table 1 in terms of Precision and Recall. Improved retrieval performance is achieved for convex curved boundary shapes reaching about 90 to $100 \%$, for concave curved boundary shapes like duck; deer shapes retrieval performance reaching between 70 and $75 \%$. In this work, retrieval performance of the proposed (skl-sig) method is compared with polar raster sampling (PRS) and area-ratio (AR) methods [20]; it is observed that the proposed method achieved improved retrieval performance over these two shape descriptors.

The future work will focus on improved retrieval performance of concave curved boundary shapes by exploring improved shape signatures. The proposed shape signatures will also be attempted and explored for shape classification. Performance evaluation with different distance metrics on proposed shape signatures will also be explored to seek improvement in retrieval time.

\section{REFERENCES}

[1] Bai,X.,ang,X., Yu,D..Latecti,L.J., 2008.SkeletonBased Shape Classification Using Path similarity, International Journal of Pattern Recognition and Artificial Intelligence, Vol.22, No.4, 733-746.

[2] Chan, T.F., Vase, L.A., 2001.Active Contours without Edges, IEEE Transactions on Image Processing, Vol.10, No.2, pp266-277.

[3] Choi,W.P., Lam,K.M., Siu,W.C.,2000. an Efficient and Accurate Algorithm for Extracting a Skeleton, IEEE Xplore .

[4] Frome,A., Singer,Y., Malik,J., 2007.Image Retrieval and Classification Using Local Distance functions, Advances in Neural Information Processing,Citeseer.

[5] Ge,Y., Fitzpatrick,J,M.,1996.On the Generation of Skeleton from Discrete Euclidean Distance Maps, IEEE Transactions on pattern analysis \& Machine Intelligence 18(11), pp.1055-1066.

[6] LandreJ., Truchetet,F., 2007.Image Retrieval with Binary Hamming Distance, Conference on Computer vision Theory, Citeseer.

[7] Malladi,R..,Sethian,j.A.,Vemuri,B.C.,1995.Shape modeling with front propagation: A level set approach, IEEE Trans. Pattern Anal. Mach. Intell.Vol.17, No.2, pp.158-175.
[8] Mercimek,D., K.Gulez,K., Velimumcu,T., 2005. Real object recognition using moment invariants, pp.765775 .

[9] Montanari, 1968. A Method for Obtaining Skeleton from Digitized Images Using a Quari- Euclidean Distance, Journal of the Association for Computing Machinary, 16(4):534-549.

[10] Mortara,M.,Patane,G.,2002.Shape-Covering for Skeleton Extraction, International Journal of Shape Modeling, Vol.8, No.2, 139-158.

[11] Nandagopalan,S.,Adiga,Dr.B.S.,deepakN.,2009.A Universal model for Content-Based Image retrieval, International Journal of computer science 4:4.

[12] Sandeep,V.M., Subhash,S.K., 2006.Curve Invariant Fast Distance Mapping Technique for Level Sets, IEEE's ICSIP 2006, Hubli, India, 777-780.

[13] Sandeep,V.M., Subhash,s.k., 2008.Hierarchical Perceptual Segmentation using arbitrary n-phase level sets, International Journal of Applied Engineering Research, ISSN 0973-4562, 3(11): 1615-1626.

[14] Sandeep,V.M., Vinayadath Kohir., Subhash kulkarni., 2010.Level set Issues for Efficient Image Segmentation, International Journal on Image and Data Fusion, ISSN.1947-9832, 1-18, 2010.

[15] Sandeep, V.M., Narayana, M., Subhash, S.K., 2008. A Scale and Rotation Invariant Fast Image Mining for Shapes, IEEE Conference on AI TOOLS in Engineering, 6-8.

[16] Sunder,H.,Silver,D., $\quad$ N.Gagvani,D.,Dickinson,S., 2003.Skeleton Based Shape Matching and Retrieval, Proceedings of the Shape Modeling International

[17] Tari,Z.S.G., Shah,J., Pienh.,1997. Extraction of Shape Skeletons from Grayscale Images, Computer Vision \& Image Understanding, Citeseer.

[18] Xiaofeng,S.,sherrill-Mix,A., Qigang Gao.,2007. Perceptual Shape-Based Natural Image Retrieval Representation and Retrieva, International Conference on Semantic Computing (ICSC).

[19] Zhang,D., Lu,G.,2001. Content-Based shape retrieval Using Different Shape Descriptors: A Comparative Stud, In Proc. Of IEEE Conference of Multimedia and Expo (ICME'01), Tokyo, August, pp.317-320.

[20] Zhang,D.,Yi Lim,M.C.,2007. An Efficient and Robust Technique for Region Based shape Representation and Retrieval, $6^{\text {th }}$ IEEE/ACIS International Conference on Computer and Information Science (ICIS). 